\title{
Detection and Localization of Iris Features for Person Identification using Digital Image Processing Techniques and Pattern Recognition Approach
}

\author{
Ashwini Chate \\ Shri Jagdishprasad Jhabarmal \\ Tibrewala University, Rajasthan, \\ India
}

\author{
Pramod Kumar \\ Shri Jagdishprasad Jhabarmal \\ Tibrewala University, Rajasthan, \\ India
}

\author{
Sushilkumar Holambe \\ Shri Jagdishprasad Jhabarmal \\ Tibrewala University, Rajasthan, \\ India
}

\begin{abstract}
Biometrics Systems are computerized methods for verification or recognition to the identity of a living person on the basis of some physiological characteristics. Human eyes are completely unique and can be used for biometric purposes. In the proposed research work, extraction of iris is done by using digital image processing techniques. For experimentation and implementation, a dataset of IIT Delhi, UBIRIS and UPOL iris image database has been used and the evaluation results clearly demonstrate that the proposed iris segmentation technique provides better accuracy in iris recognition rather than existing techniques. Classification is done by using supervised and unsupervised pattern classification techniques. Support vector machine and k-means clustering is used for classification iris features. Overall got $97.25 \%$ accuracy based on confusion matrix. The results clearly prove that the proposed technique provides better, rather than existing techniques.
\end{abstract}

\section{General Terms}

Iris localization is done by using DIP techniques and classification is performed by using supervised and unsupervised learning.

\section{Keywords}

Iris Detection, Digital Image Processing, Pattern Classification, Supervised Learning, Unsupervised Learning.

\section{INTRODUCTION}

Biometric Systems are computerized methods for verification or recognition to the identity of a living person on the basis of some physiological characteristics, like a fingerprint or face pattern or some aspects of behavior, like handwriting or keystroke patterns. A biometric system based on physiological characteristics is more dependable than one which adopts behavioral features, even if the latter may be easier to integrate within certain specific applications. The iris and the retina as elements inside the eye are very well protected against damage.The iris and retina patterns are unique to every person also applies to monozygotic twins, although the structure and iris color are genetically dependent. Following figure shows the anatomy of human eye. In anatomy of human eye, there are different parts such as, iris, pupil, cornea, sclera, lens, calliary body retina, optic nerve, choroid and so on.Iris recognition is an automated method of biometric identification that uses mathematical pattern-recognition techniques on images of one or both of the irises of an individual's eyes, whose complex patterns are unique, steady, and can be seen from some distance.

\section{LITERATURE REVIEW}

An accurate biometric identification system is a critical requirement in a variety of applications. Iris-based identification has been receiving a lot of attention since its introduction. However, some techniques have limitations in identifying persons accurately and efficiently. Local and global characteristics of the iris, using a band of log-Gabor filter is used, vector that stores these characteristics a feature descriptor or simply the iris descriptor [1].Biometric authentication system based on the fusion of two user-friendly biometric modalities: Iris and Face. Using one biometric feature can lead to good results, but there is no reliable way to verify the classification. In order to reach robust identification and verification we are combining two different biometric features. we specifically apply 2-D discrete wavelet transform to extract the feature sets of low dimensionality from iris and face. And then to obtain Reduced Joint Feature Vector (RJFV) from these feature sets, Direct Linear Discriminant Analysis (DLDA) is used in our multimodal system. This system can operate in two modes: to identify a particular person or to verify a person's claimed identity. Results for both cases show that the method leads to a reliable person authentication system [2].With an increasing emphasis on security, automated personal identification based on biometrics has been receiving extensive attention over the 1990s. Iris recognition, as an emerging biometric recognition approach is becoming a very active topic in both research and practical applications. Iris recognition is the process of recognizing a person by analyzing the apparent pattern of his or her iris. A typical iris recognition system includes iris imaging, iris detection, feature extraction and recognition. Existing techniques utilized at the different stages of the iris recognition process and evaluate their performance. In addition, we explore additional techniques to improve performance [3].In the absence of real data for extensive testing of newly designed large-scale biometrics recognition systems a number of solutions are possible including use of resampling methods, generation of synthetic data having properties similar to real data of interest, or use of analytical tools to predict the performance. Each of the methods has its own limitations. In this work, we focus on iris biometric. We briefly describe a model based approach to synthesize iris images and focus on performance comparison for synthesized and real iris images. Iris image processing assumes a traditional Gabor 
filter based encoding approach [4].Most biometric identification methods using iris recognition assume that the eye image of the person is available, and are only concerned with the extraction of the iris from the eye image. These methods require the person to look directly into the camera, which is a rather uncomfortable process. Robust approach is to initially locate eye regions within facial images taken from a distance. Then the iris image is extracted from the detected regions. Hence, a nonintrusive and more comfortable iris imaging approach has been implemented [5].A biometric system provides automatic identification of an individual based on a unique feature or characteristic possessed by the individual. Iris recognition is regarded as the most reliable and accurate biometric identification system available. The iris recognition system consists of an automatic segmentation system that is based on the Hough transform, and is able to localize the circular iris and pupil region, occluding eyelids and eyelashes, and reflections. The extracted iris region was then normalized into a rectangular block with constant dimensions to account for imaging inconsistencies. Finally, the phase data from 1D Log-Gabor filters was extracted and quantized to four levels to encode the unique pattern of the iris into a bit-wise biometric template. The Hamming distance was employed for classification of iris templates, and two templates were found to match if a test of statistical independence was failed [6].Iris identification (IRI) constitutes an increasingly accepted methodology of biometrics. IRI is based on the successful encoding and matching of distinctive iris features (folds, freckles etc.), which - in turn - presupposes that iris segmentation has been accurately performed. In contrast to the inner (iris/pupil) iris boundary, which - owing to the high contrast between the adjacent areas - is relatively easy to localize, detection of the outer (iris/sclera) iris boundary is more challenging since the low contrast between the separated areas often results in fragmented, ambiguous and spurious edges. A novel approach to iris boundary detection is presented here, featuring a genetic algorithm (GA) for outer iris boundary detection [7].A new and efficient method for combining iris biometrics with custom cryptographic schemes to obtain an efficient biocryptosystem is developed. Though the method structure is basically derived from a previously described biocryptosystem scheme, the introduction of new image processing methods alongside with efficient utilization of traditional methods show promising developments compared with the previous biocryposystem especially in the field of generating longer cryptographic key strings while keeping the system quality [8].Crypto-biometric systems have been studied for solving the key management problem of cryptographic systems and protecting templates in biometric systems at the same time. The fuzzy vault system is a well-known cryptobiometric system. We propose a new method of applying iris data to the fuzzy vault. Our research has following two advantages and contributions. First, in order to solve the variation problem of the extracted iris features, we introduce a pattern clustering method. Second, in order to produce unordered sets for fuzzy vault, we use the iris feature extraction algorithm based on ICA (Independent Component Analysis). Experimental results showed that 128-bit cryptographic keys as well as the iris templates were secure with the fuzzy vault scheme [9].A new iris recognition system based on complex wavelet Transforms is described. In this work iris recognition based on
Gabor wavelet and Morlet wavelet are described. The most unique phenotypic feature visible in a person's face is the detailed texture of each eye's iris. The visible texture of a person's iris is encoded into a compact sequence of 2-D wavelet coefficients, which generate an ldquoiris coderdquo of 4096-bits. The statistical parameters like mean and covariance of coefficients of the iris images are also computed. Two different iris codes are compared using exclusively OR comparisons. Also the new iris pattern is compared against the stored pattern after computing the probability of new iris pattern and identification is performed [10].New combination technique to fuse scores deriving from face and iris biometric matchers. Based on a precise statistical analysis of bootstrapped match scores deriving from similarity matrices, we show the utility of wavelet denoising on normalized scores. Then, we use an adaptive fusion rule based on the maximization of a cost function combining user-specific weights, a separation distance and statistical moments. Experiments are conducted on FERET and CASIA databases and results show that method outperforms by $70 \%$ some of the best current combination approaches in terms of Equal Error Rates (EER), and reaches a Genuine Accept Rate (GAR) equals to $100 \%$ at a False Accept Rate (FAR) of $7 \times 10-4 \%$ [11].Iris recognition is mostly used in biometrics for personal identification. It is the most powerful tool for person identification. But in real time it is quite difficult to capture the better quality of iris images. The images obtained are more degraded due to the lack of texture, blur. A simple high instability technique is presented also this process is more convenient to use. This super-resolution algorithm is applied to the pixels of iris images to select the best frame from the iris image. A segmentation algorithm that segments the input iris images. Spatial FCM used for segmentation testing purpose. Iris image is framing $600 \mathrm{x}$ 600 in sequence to calculate vessel area in each framework using for pattern abstraction using loop descriptor. The Hough transforms cast-off de-noising the image. The experimental results show that system successfully recognizes the iris about 4 to 8 meters long distance of a person. Developed on MATLAB for "reading" the profile also for completing the Hough transforms performance. The database used for this purpose is CASIA V4. The simulation results show that the stable extraction of iris recognition [12]. The widespread interest in personal identification has increased the need for an accurate and efficient ways of identification, verification and authentication. Biometricbased personal identification systems have proven their superior performance. These systems rely on the person physiological traits such as the iris, fingerprint or face, etc. It is worth noting that these systems are more effective than conventional personal identification systems that are based on passwords and/or smartcards. In recent years, there has been emergence in the fusion/combination of multi-biometric traits to further enhance the performance of biometric systems. A systematic pattern retrieval algorithm is applied in order to improve the accuracy of overall system. Normalized iris sub-images are fused based on a specific quality measure. Simulation results clearly indicate the improvement in performance due to the iris fusion scheme [13]. 


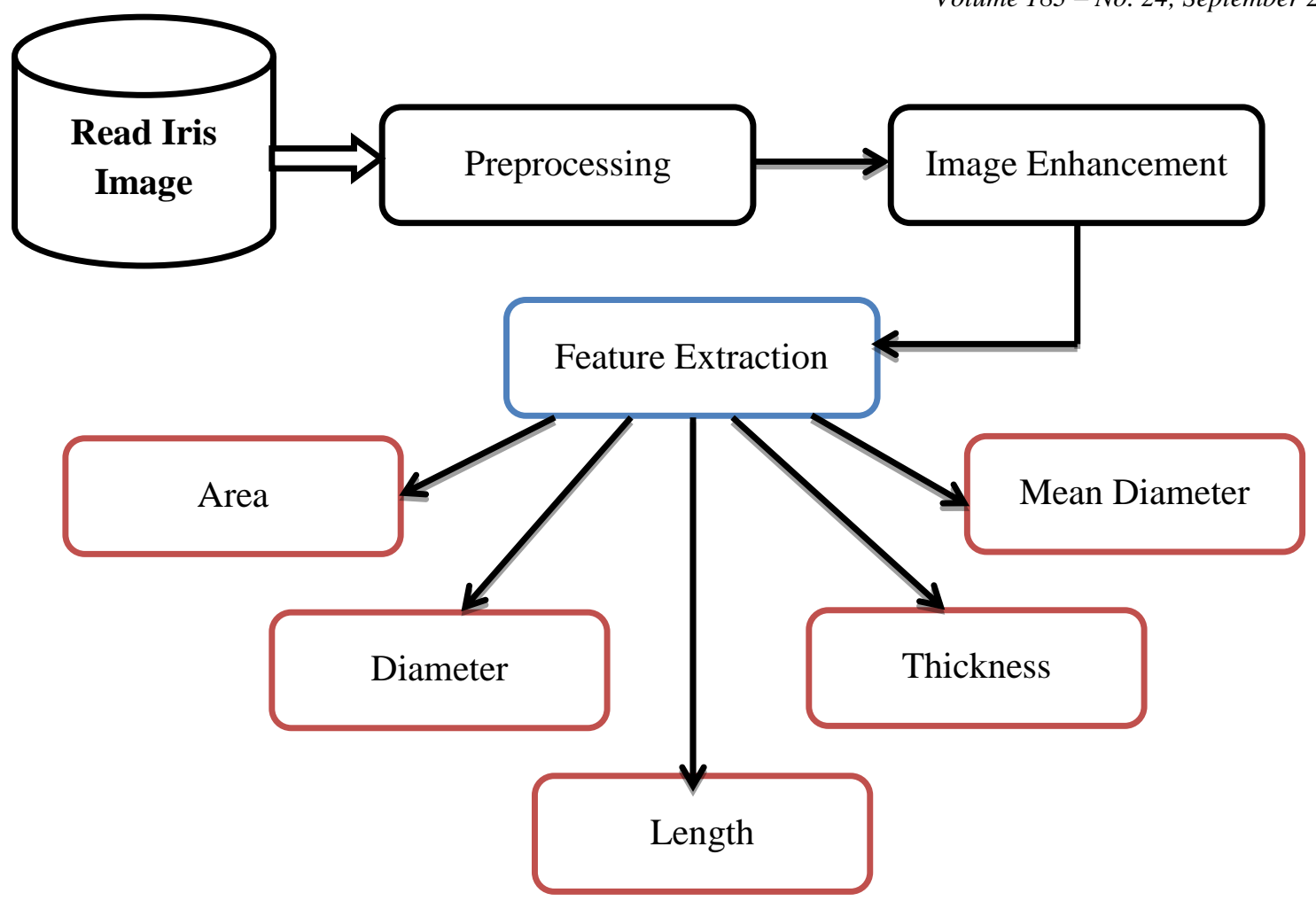

Fig. 1: Workflow of Detection and Localization of Iris Features

\section{METHODOLOGY}

Iris recognition is the most accurate form of biometric identification. The robustness of iris recognition comes from the unique characteristics of the human iris texture as it is stable over the human life, and the environmental effects cannot easily alter its shape. Above figure shows the workflow for detection and localization of iris features. First resizing all the iris image into one dimension and second convert all 3D images into 2D. Afterwards image enhancement is done by using digital image processing techniques. Subsequently apply quality measures for the better performance of the algorithm. Feature extraction is done by using iris localization. Following are mathematical formulation.

\subsection{Conversion of 3D to 2D}

Average method is the simplest one to convert 3D image into 2D. Just have to take the average of three colors. Since it's an RGB image, so it means that we have added $r$ with $g$ with $b$ and then divide it by 3 to get your desired 2D image.

$$
\operatorname{grayscale}(2 D)=\frac{R+G+B}{3}
$$

\subsection{Image Enhancement}

Image enhancement techniques have been widely used in many applications of image processing where the subjective quality of images is important for human interpretation. Contrast is an important factor in any subjective evaluation of image quality. Contrast is created by the difference in luminance reflected from two adjacent surfaces. In other words, contrast is the difference in visual properties that makes an object distinguishable from other objects and the background.

$$
G(j, k)=T[F(j, k)]
$$

Where, $F(j, k)$ is the input image $G(j, k)$ is the output image and $\mathrm{T}$ is operator defined over a neighborhood.

\subsection{Feature Extraction}

Feature extraction is a type of dimensionality reduction where a large number of pixels of the image are efficiently represented in such a way that interesting parts of the image are captured effectively. The first step in feature extraction is defining edges that can be found in the image and attempting to combine these into objects and shapes that are assumed to be in the image. After segmentation of iris images, feature extraction is performed by computing the area, length, thickness, diameter and mean diameter.

Area

$$
\text { Area }=\pi \times r^{2}(3)
$$

Diameter

$$
\text { Diameter }=\sqrt{\text { Area } / \pi}(4)
$$

Length

$$
\text { Length }=\frac{\text { Area }}{2}(5)
$$

Thickness

$$
\text { Thickness }=\frac{\text { Area }}{\text { Length }}(6)
$$

Mean Diameter

$$
\text { Mean }=\frac{\sum X}{n}(7)
$$

\section{RESULT}

For experimentation and implementation, a dataset of IIT Delhi, UBIRS and UPOL iris image database has been used and the evaluation results clearly demonstrate that the proposed iris segmentation technique provides better accuracy 
in iris recognition rather than existing techniques. After collection of databases, preprocessing is done into two ways. First resizing all the iris image into one dimension and second convert all 3D images into 2D. Afterwards image enhancement is done by using digital image processing techniques. Subsequently apply quality measures for the better performance of the algorithm. Feature extraction is done by using iris localization. Also design graphical user interface for the proposed algorithm. Finally classification is done by using supervised and unsupervised pattern classification techniques. Support vector machine and k-means clustering is used for classification iris feature.Identification of person based on iris is done by computing the statistical features of iris. The features are area, length, thickness, diameter, and mean diameter of extracted iris. Based on these statistical features we can identify the person. Following tables shows the output of statistical features.

Table 1.Statistical Features of IIT Delhi Iris Image Database

\begin{tabular}{|c|l|l|l|l|l|}
\hline $\begin{array}{c}\text { Sr. } \\
\text { No }\end{array}$ & Area & Diameter & Length & Thickness & $\begin{array}{c}\text { Mean } \\
\text { Diameter }\end{array}$ \\
\hline $\mathbf{1}$ & 3208 & 180 & 1604 & 2.00 & 20.00 \\
\hline $\mathbf{2}$ & 5291 & 232 & 2646 & 1.95 & 19.98 \\
\hline $\mathbf{3}$ & 7223 & 271 & 3612 & 1.93 & 19.97 \\
\hline $\mathbf{4}$ & 2605 & 162 & 1303 & 1.95 & 19.94 \\
\hline $\mathbf{5}$ & 6599 & 259 & 3300 & 1.97 & 19.98 \\
\hline $\mathbf{6}$ & 4515 & 214 & 2258 & 1.98 & 19.94 \\
\hline $\mathbf{7}$ & 1858 & 137 & 929 & 1.99 & 20.00 \\
\hline $\mathbf{8}$ & 5489 & 236 & 2745 & 1.95 & 19.92 \\
\hline $\mathbf{9}$ & 5822 & 243 & 2911 & 1.97 & 20.00 \\
\hline $\mathbf{1 0}$ & 2291 & 152 & 1146 & 1.99 & 19.92 \\
\hline $\mathbf{1 1}$ & 7215 & 270 & 3608 & 1.97 & 19.98 \\
\hline $\mathbf{1 2}$ & 3092 & 177 & 1546 & 1.97 & 19.98 \\
\hline $\mathbf{1 3}$ & 2000 & 142 & 1000 & 1.98 & 19.94 \\
\hline $\mathbf{1 4}$ & 5740 & 241 & 2870 & 1.99 & 20.00 \\
\hline $\mathbf{1 5}$ & 5719 & 241 & 2860 & 1.95 & 19.92 \\
\hline $\mathbf{1 6}$ & 5865 & 244 & 2933 & 1.97 & 20.00 \\
\hline $\mathbf{1 7}$ & 2450 & 158 & 1225 & 1.99 & 19.92 \\
\hline $\mathbf{1 8}$ & 4607 & 216 & 2304 & 1.97 & 19.98 \\
\hline $\mathbf{1 9}$ & 3836 & 197 & 1918 & 1.98 & 19.94 \\
\hline $\mathbf{2 0}$ & 4902 & 223 & 2451 & 1.99 & 20.00 \\
\hline $\mathbf{2 1}$ & 6568 & 258 & 3284 & 1.95 & 19.92 \\
\hline $\mathbf{2 2}$ & 2519 & 160 & 1260 & 1.97 & 20.00 \\
\hline $\mathbf{2 3}$ & 9455 & 310 & 4728 & 1.99 & 19.92 \\
\hline $\mathbf{2 4}$ & 5369 & 233 & 2685 & 1.97 & 19.98 \\
\hline $\mathbf{2 5}$ & 4508 & 214 & 2254 & 1.98 & 19.94 \\
\hline $\mathbf{2 6}$ & 5822 & 243 & 2911 & 1.97 & 20.00 \\
\hline $\mathbf{2 7}$ & 2291 & 152 & 1146 & 1.99 & 19.92 \\
\hline $\mathbf{2 8}$ & 7215 & 270 & 3608 & 1.97 & 19.98 \\
\hline $\mathbf{2 9}$ & 3092 & 177 & 1546 & 1.97 & 19.98 \\
\hline $\mathbf{3 0}$ & 2000 & 142 & 1000 & 1.98 & 19.94 \\
\hline $\mathbf{3 1}$ & 5740 & 241 & 2870 & 1.99 & 20.00 \\
\hline $\mathbf{3 2}$ & 5719 & 241 & 2860 & 1.95 & 19.92 \\
\hline $\mathbf{3 3}$ & 2291 & 152 & 1146 & 1.99 & 19.92 \\
\hline $\mathbf{3 4}$ & 7215 & 270 & 3608 & 1.97 & 19.98 \\
\hline $\mathbf{3 5}$ & 3092 & 177 & 1546 & 1.97 & 19.98 \\
\hline & & & & & \\
\hline
\end{tabular}

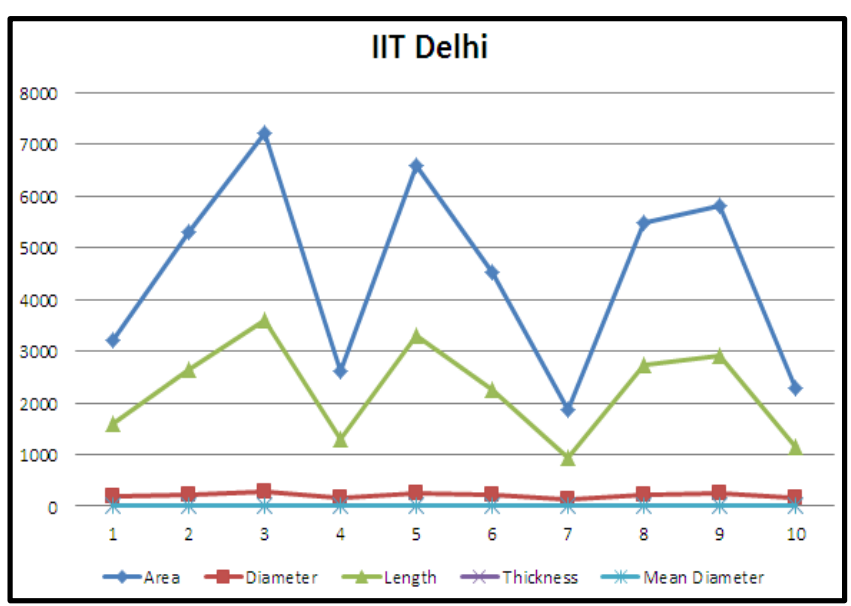

Fig. 2: Statistical Features of IIT Delhi Iris Image Database

Table 2. Statistical Features of UBIRIS Iris Image Database

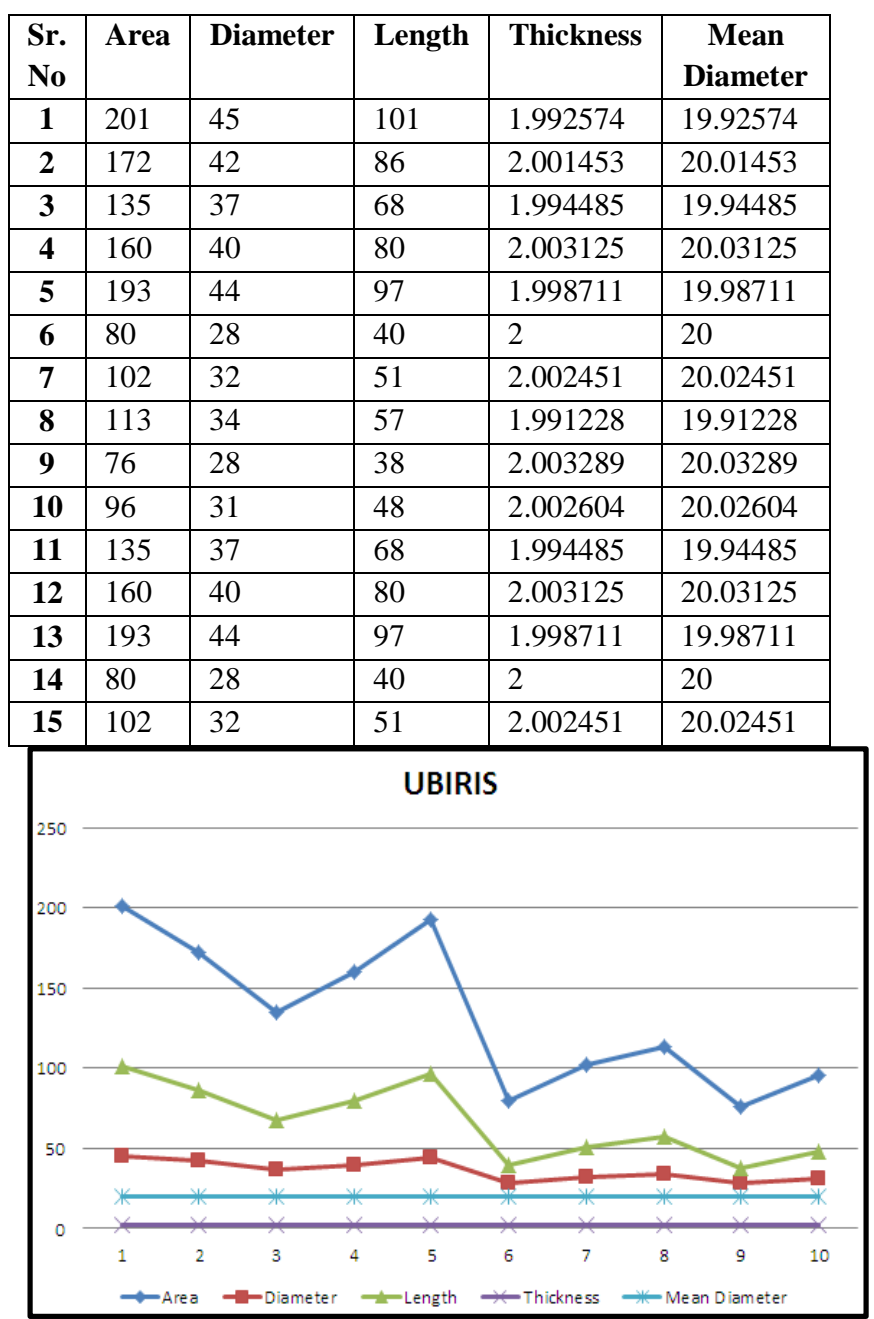

Fig. 3: Statistical Features of UBIRIS Iris Image Database 
Table 3. Statistical Features of UPOL Iris Image Database

\begin{tabular}{|c|l|l|l|l|l|}
\hline $\begin{array}{c}\text { Sr. } \\
\text { No }\end{array}$ & Area & Diameter & Length & Thickness & $\begin{array}{c}\text { Mean } \\
\text { Diameter }\end{array}$ \\
\hline $\mathbf{1}$ & 135189 & 1170 & 67595 & 1.92 & 19.93 \\
\hline $\mathbf{2}$ & 133057 & 1161 & 66529 & 1.94 & 19.23 \\
\hline $\mathbf{3}$ & 135026 & 1170 & 67513 & 2.00 & 20.01 \\
\hline $\mathbf{4}$ & 132266 & 1158 & 66133 & 2.10 & 20.05 \\
\hline $\mathbf{5}$ & 132712 & 1160 & 66356 & 2.12 & 20.09 \\
\hline $\mathbf{6}$ & 132330 & 1158 & 66165 & 2.23 & 20.15 \\
\hline $\mathbf{7}$ & 131260 & 1153 & 65630 & 2.33 & 20.14 \\
\hline $\mathbf{8}$ & 130645 & 1151 & 65323 & 1.97 & 19.97 \\
\hline $\mathbf{9}$ & 131699 & 1155 & 65850 & 1.98 & 19.92 \\
\hline $\mathbf{1 0}$ & 13046 & 1150 & 65231 & 1.97 & 19.94 \\
\hline
\end{tabular}

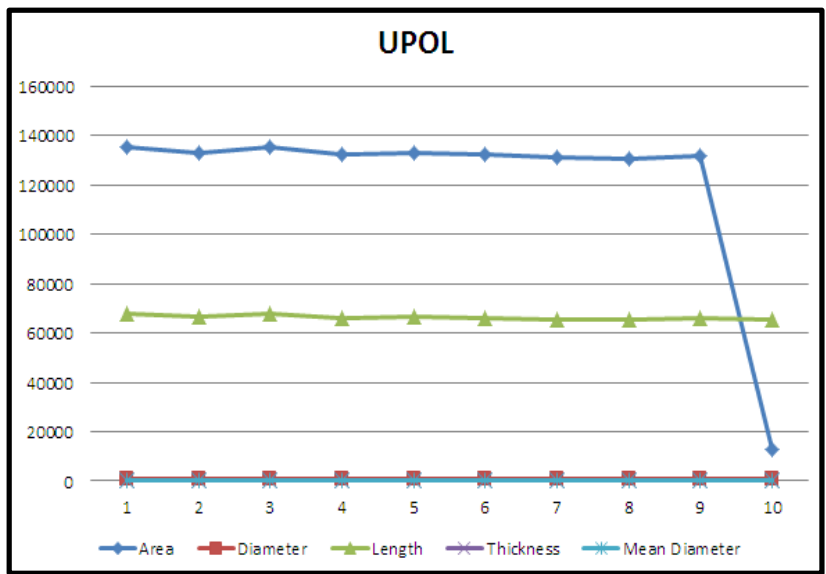

Fig.4: Statistical Features of UPOL Iris Image Database

\subsection{K-Means Clustering}

K-Means clustering uses a two-phase iterative algorithm to minimize the sum of point-to-centroid distances, summed over all $\mathrm{k}$ clusters. Here we pass the area, length, thickness, diameter and mean diameter of extracted iris images. Following figure shows the output of K-Means clustering.

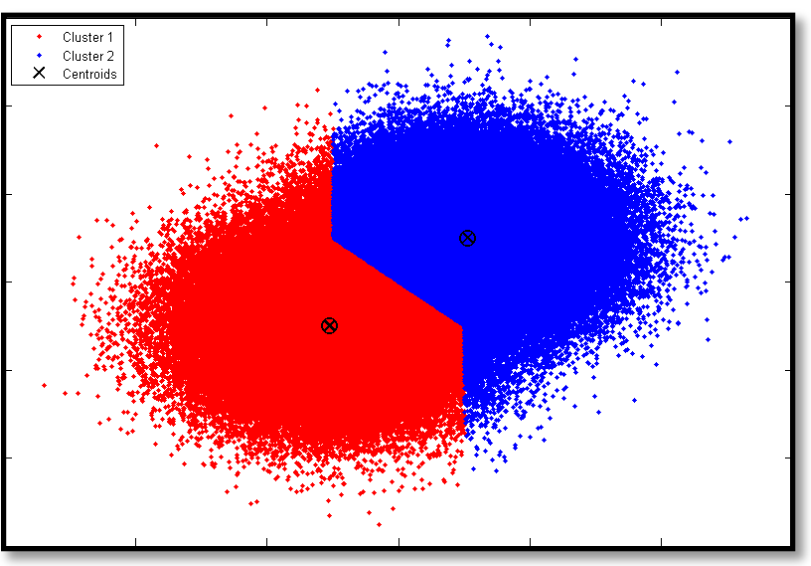

Fig. 5: K-Means Clustering

\subsection{Supoort Vector Machine (SVM)}

Support Vector Machine (SVM) is a supervised machine learning algorithm which used for both classification and regression challenges. SVM is widely used for classification problems. We apply the SVM classifier to iris feature set. Following figure shows the output of SVM classification.

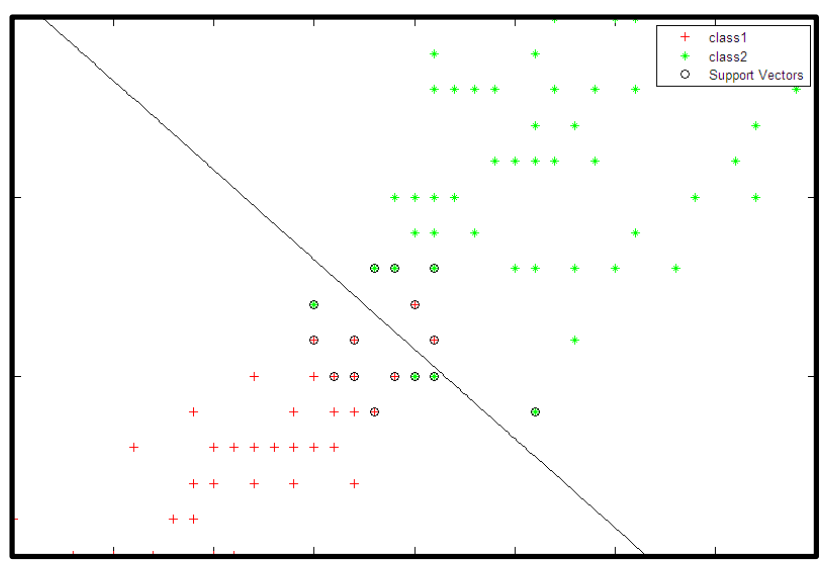

Fig. 6. Support Vector Machine (SVM)

\subsection{Performance Analysis using ROC}

The receiver operating characteristic (ROC) curve is a graphical plot which shows the performance of a classifier system as its discrimination threshold is varied. The curve is created by plotting the true positive rate against the false positive rate at various threshold settings.

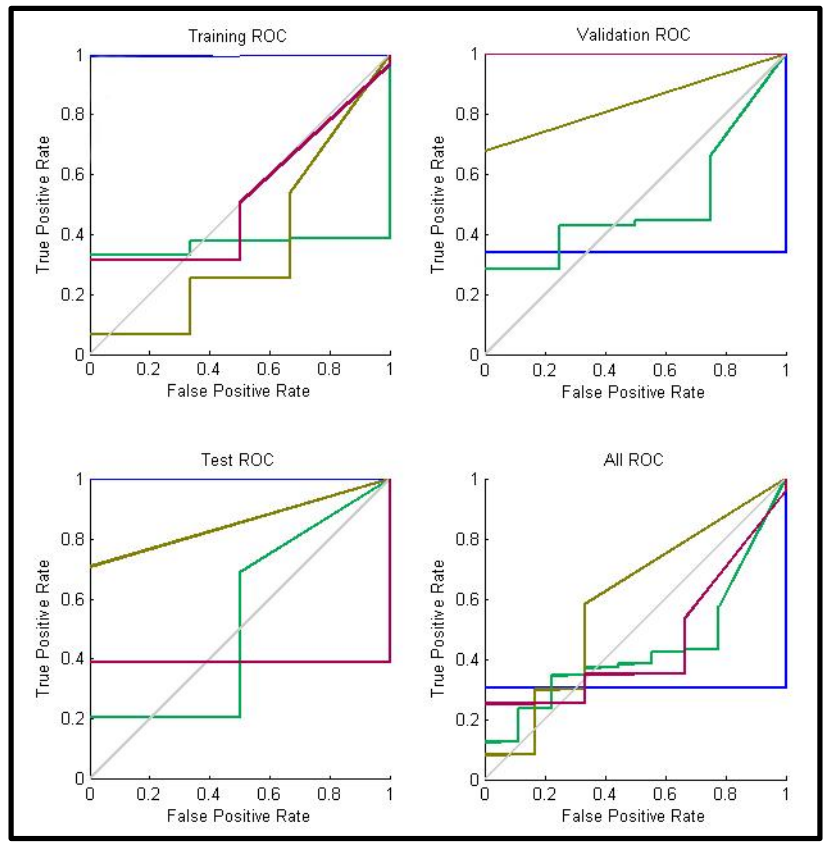

Fig. 7: Receiver Operating Characteristic (ROC) Curve

\section{CONCLUSION}

Biometric techniques, which recognize individual's dependent on physical or behavioral traits, are of importance since individuals can't fail to remember or lose their a physical characteristics in the manner that they can lose passwords or identity cards. Among these biometric approaches, iris is right now considered as one of the most solid biometrics due to its remarkable 
surface's arbitrary variety. Additionally, iris is end up being all around shielded from the outside climate behind the cornea, moderately simple to secure and stable everywhere on the individual's life. Implement this algorithm on open source iris databases (IIT Delhi, UBIRIS and UPOL Iris Database). A statistical feature extraction has been proposed and implemented in this work. It is demonstrated that statistical features can be computed using area, diameter, length, thickenss and mean diameter of iris image. System performance in both the directions is satisfactory. Experimental results obtained on statistical feature extraction technique are encouraging. It has been seen that system performance is improved with increased number of statistical features, supervised and unsupervised learning.

\section{ACKNOWLEDGMENTS}

We are thankful to IIT Delhi, UBIRIS and UPOL for providing iris image databases.

\section{REFERENCES}

[1] Seif, R. Zewail, M. Saeb and N. Hamdy (2003), "Iris identification based on log Gabor filtering," 46th Midwest Symposium on Circuits and Systems, Cairo, 2003, pp. 333-336 Vol. 1, doi: 10.1109/MWSCAS.2003.1562286.

[2] Son B., Lee Y. (2005) Biometric Authentication System Using Reduced Joint Feature Vector of Iris and Face. In: Kanade T., Jain A., Ratha N.K. (eds) Audio- and Video-Based Biometric Person Authentication. AVBPA 2005. Lecture Notes in Computer Science, vol 3546. Springer, Berlin, Heidelberg. https://doi.org/10.1007/11527923_53.

[3] [3] S. M. Elsherief, M. E. Allam and M. W. Fakhr (2006), "Biometric Personal Identification Based on Iris Recognition," 2006 International Conference on Computer Engineering and Systems, Cairo, 2006, pp. 208-213, doi: 10.1109/ICCES.2006.320449.

[4] J. Zuo, N. A. Schmid and X. Chen (2006), "On Performance Comparison of Real and Synthetic Iris Images," 2006 International Conference on Image Processing, Atlanta, GA, 2006, pp. 305-308, doi: 10.1109/ICIP.2006.313154.

[5] Erturk, S. (2006). Nonintrusive Iris Image Extraction for Iris Recognition-Based Biometric Identification. Circuits Syst Signal Process 25, 405-419. https://doi.org/10.1007/s00034-005-0305-6.

[6] P. Padma Polash and M. M. Monwar (2007), "Human iris recognition for biometric identification," 2007 10th international conference on computer and information technology, Dhaka, 2007, pp. 1-5, doi: 10.1109/ICCITECHN.2007.4579354.

[7] Tambouratzis T., Masouris M. (2007) GA-Based Iris/Sclera Boundary Detection for Biometric Iris Identification. In: Beliczynski B., Dzielinski A., Iwanowski M., Ribeiro B. (eds) Adaptive andNatural Computing Algorithms. ICANNGA 2007. Lecture Notes in Computer Science, vol 4432. Springer, Berlin, Heidelberg. https://doi.org/10.1007/978-3-54071629-7_52.

[8] Shojaee Bakhtiari A., Beheshti Shirazi A.A., Zamanlooy B. (2007) An Efficient Biocryptosystem Based on the Iris Biometrics. In: Mery D., Rueda L. (eds) Advances in Image and Video Technology. PSIVT 2007. Lecture Notes in Computer Science, vol 4872. Springer, Berlin, Heidelberg. https://doi.org/10.1007/978-3-540-77129-6_31.

[9] Lee Y.J., Bae K., Lee S.J., Park K.R., Kim J. (2007) Biometric Key Binding: Fuzzy Vault Based on Iris Images. In: Lee SW., Li S.Z. (eds) Advances in Biometrics. ICB 2007. Lecture Notes in Computer Science, vol 4642. Springer, Berlin, Heidelberg. https://doi.org/10.1007/978-3-540-745495_84.

[10] S. Hariprasath and V. Mohan (2008), "Biometric personal identification based on iris recognition using complex wavelet transforms," 2008 International Conference on Computing, Communication and Networking, St. Thomas, VI, 2008, pp. 1-5, doi: 10.1109/ICCCNET.2008.4787736.

[11] Morizet N., Gilles J. (2008) A New Adaptive Combination Approach to Score Level Fusion for Face and Iris Biometrics Combining Wavelets and Statistical Moments. In: Bebis G. et al. (eds) Advances in Visual Computing. ISVC 2008. Lecture Notes in Computer Science, vol 5359. Springer, Berlin, Heidelberg. https://doi.org/10.1007/978-3-54089646-3 65.

[12] S. D. Shirke and C. Rajabhushnam (2009), "Biometric Personal Iris Recognition from an Image at Long Distance," 2019 3rd International Conference on Trends in Electronics and Informatics (ICOEI), Tirunelveli, India, 2019, pp. 560-565, doi: 10.1109/ICOEI.2019.8862640.

[13] A. A. Bahjat and L. Ghouti (2009), "Quality based iris recognition," 3rd International Conference on Imaging for Crime Detection and Prevention (ICDP 2009), London, 2009, pp. 1-6, doi: 10.1049/ic.2009.0248

[14] IIT Delhi Iris database. (2020). Iris Image Database. https://www4.comp.polyu.edu.hk/ csajaykr/IITD/Databa se_Iris.htm [Accessed 20 September 2020].

[15] UBIRIS Version 1 database. (2020). Iris Image Database. https://www http://iris.di.ubi.pt/ [Accessed 21 September 2020].

[16] University-of-Palackeho-and-Olomouc.(2020). UPOL Iris Database [Online]. Available: http://phoenix.inf.upol.cz/iris/. [Accessed 23 September 2020].

[17] S. Gopi (2020) Human Iris using Neural Network, International Journal of Engineering Research \& Technology (IJERT) ISSN: 2278-0181.

[18] Juan Wang(2020) An Improved Iris Recognition Algorithm Based on Hybrid Feature and ELM, IOP Conference Series: Materials Science and Engineering, Volume 322, Issue 5.

[19] Arora, S., Bhatia, M.P.S. (2020) Presentation attack detection for iris recognition using deep learning. International Journal Syst Assur Eng Manag 11, 232-238 (2020). https://doi.org/10.1007/s13198-02000948-1.

[20] S. D. Thepade and R. K. Bhondave (2015), "Multimodal identification technique using Iris \& 
Palmprint traits with matching score level in various Color Spaces with BTC of bit plane slices," 2015 International Conference on Industrial Instrumentation and Control (ICIC), Pune, 2015, pp. 1469-1473, doi: 10.1109/IIC.2015.7150981.

[21] A. Varshney, A. Rani and V. Singh (2015), "Optimization of filter parameters for iris detection," 2015 4th International Conference on Reliability, Infocom Technologies and Optimization (ICRITO) (Trends and Future Directions), Noida, 2015, pp. 16, doi: 10.1109/ICRITO.2015.7359369.

[22] D. Menotti et al (2015), "Deep Representations for Iris, Face, and Fingerprint Spoofing Detection," in IEEE Transactions on Information Forensics and Security, vol. 10, no. 4, pp. 864-879, April 2015, doi: $10.1109 /$ TIFS.2015.2398817.

[23] Pournima Ghanmode, Snehal Mahajan2 , Pragati Ghodake , Babita Sonare (2016) Iris Recognition Based on Extreme Point Identification using Feature Extraction, International Journal of Advanced Research in Computer and Communication Engineering Vol. 5, Issue 4.

[24] Jyoti poonia, Parvati Bhurani, Sandeep Kumar Gupta, Shubh Lakshmi Agrwal (2016) New Improved Feature Extraction Approach of IRIS Recognition, International Journal of Computer Systems (ISSN: 2394-1065), Volume 03- Issue 01.

[25] Sarita G.Firake, P.M. Mahajan (2016) Comparison of
Iris Recognition Using Gabor Wavelet, Principal Component Analysis and Independent Component Analysis, International Journal of Innovative Research in Computer and Communication Engineering, ISSN(Online): 2320-9801, Vol. 4, Issue 6.

[26] Manasa G, Prathusha J S, Supriya Y N, Saritha Chakrasali (2016) Multimodal Biometrics-Feature Level Fusion of Face and Iris Biometrics, International Journal of Engineering Research \& Technology (IJERT) ISSN: 2278-0181.

[27] R. Luhadiya and A. Khedkar (2016), "Iris detection for person identification using multiclass SVM," 2016 IEEE International Conference on Advances in Electronics, Communication and Computer Technology (ICAECCT), Pune, 2016, pp. 387-392, doi: 10.1109/ICAECCT.2016.7942619.

[28] A. Matin, F. Mahmud, S. T. Zuhori and B. Sen, "Human iris as a biometric for identity verification," 2016 2nd International Conference on Electrical, Computer \& Telecommunication Engineering (ICECTE), Rajshahi, 2016, pp. 1-4, doi: 10.1109/ICECTE.2016.7879610.

[29] S. Patil, S. Gudasalamani and N. C. Iyer (2016), "A survey on Iris recognition system," 2016 International Conference on Electrical, Electronics, and Optimization Techniques (ICEEOT), Chennai, 2016, pp. 2207-2210, doi 10.1109/ICEEOT.2016.7755084 